Introduction Adverse Left ventricular (LV) remodelling with increase in LV volumes and reduction in ejection fraction (EF) can occur following ST-elevation myocardial infarction (STEMI) and confers worse prognosis. LV remodelling is mediated in part by degradation of the extra-cellular matrix, through the activity of Matrix Metalloproteinase (MMP) enzymes and their intrinsic inhibitors, the tissue inhibitors of matrix metalloproteinases (TIMPs).

We examined the relationships of NTproBNP, MMP and TIMP biomarkers measured at index admission with infarct characteristics, and relations to subsequent changes in LV volumes and mass.

Methods Seventy-five patients with first STEMI presenting between January and December 2010 were prospectively enrolled. Pre-discharge serum samples were collected (median $45 \mathrm{~h}$, IOR $40 \mathrm{~h}$ ) before analysis via in-house chemiluminescent assay. Patients underwent Cardiac Magnetic Resonance (CMR) examination (1.5 T Seimens Avanto) during admission (median day 2, IOR 2 days) incorporating volume assessment (cine-SSFP) with further follow-up volume assessment at 4 months (median 120 days, IOR 8 days) completed in 67 patients. Percentage LV remodelling was determined by the following index for changes in end-systolic and end-diastolic volumes and masses:

(Volume at 4 months-Volume at STEMI) $\div$ Volume at STEMI $\times 100 \%$

Patient demographics are shown in table 1.

Where required, data was log transformed to normalise distribution. Biomarkers were correlated to volume and mass changes using Pearson's correlation to compare continuous variables and Spearman's rank correlation to compare non-parametric data. Correlations with $p<0.1$ were entered into multivariate linear regression model to predict subsequent remodelling.

Results TIMP-4 levels sampled at the time of STEMI correlated with a relative change in LV end-systolic volume index (LVESVI) $(\mathrm{R}=0.315, \mathrm{p}=0.009)$. Microvascular obstruction $(\mathrm{MVO})$ and intramyocardial haemorrhage (IMH) also correlated with LVESVI. There were no biomarker correlates with a relative change in LV End-diastolic volume index (LVEDVI). Percentage MVO and IMH positively correlated with LVEDVI. EF at 4 months correlated significantly with MMP3 $(\mathrm{R}=-0.346, \mathrm{p}=0.004)$, NTproBNP $(\mathrm{R}=$ $-0.450, p<0.001)$, time to reperfusion, Infarct size, MVO, IMH, and myocardial salvage index (MSI). There were no significant biomarker correlates with LV mass index.

On univariate linear regression analysis, TIMP4 was an independent predictor of relative change in LVESVI $(R=0.294, t=2.483$, $\mathrm{p}=0.016$ ). TIMP4 remained the only significant predictor of ESVI change on multivariate linear regression analysis incorporating IS\%, MVO\%, IMH\% (R-0.390, TIMP4 t=2.131, p=0.037).

NTproBNP remained the only independent predictor of EF at 4 months on multivariate linear regression $(R=0.738, t=2.162$, $\mathrm{p}=0.036)$. NTproBNP was also the only significant predictor of Infarct size at STEMI $(\mathrm{R}=0.733, \mathrm{t}=4.852, \mathrm{p}<0.001)$.

Table 1

\section{RELATION OF MATRIX METALLOPROTEINASES AND THEIR TISSUE INHIBITORS TO LEFT VENTRICULAR REMODELLING FOLLOWING FIRST ST-ELEVATION MYOCARDIAL INFARCTION}

N A Razvi, ${ }^{1}$ B Grundy, ${ }^{2}$ L L Ng, ${ }^{1}$ G P McCann, ${ }^{1}$ I B Squire ${ }^{1}$ University of Leicester; ${ }^{2}$ Cardiovascular Biomedical Research Unit, NIHR

doi:10.1136/heartjn-2013-304019.13

\begin{tabular}{lc}
\hline Age (years) & $\mathbf{5 9 . 2} \pm \mathbf{1 0 . 9} \mathbf{( 9 3 \%}$ male gender) \\
\hline Reperfusion therapy: & PPCI $(58 \%)$, thrombolysis $(16 \%)$, rescue \\
& PCI (12\%), completed/late presenting infarct $(13 \%)$ \\
LVESVI at STEMI & $58.0 \pm 17.67$ \\
LVESVI at 4 months & $53.2 \pm 18.35$ \\
LVEDVI at STEMI & $95.84 \pm 18.15$ \\
LVEDVI at 4 months & $97.29 \pm 22.25$ \\
EF at STEMI & $41.17 \pm 8.35$ \\
EF at 4 months & $47.21 \pm 8.39$ \\
\hline
\end{tabular}




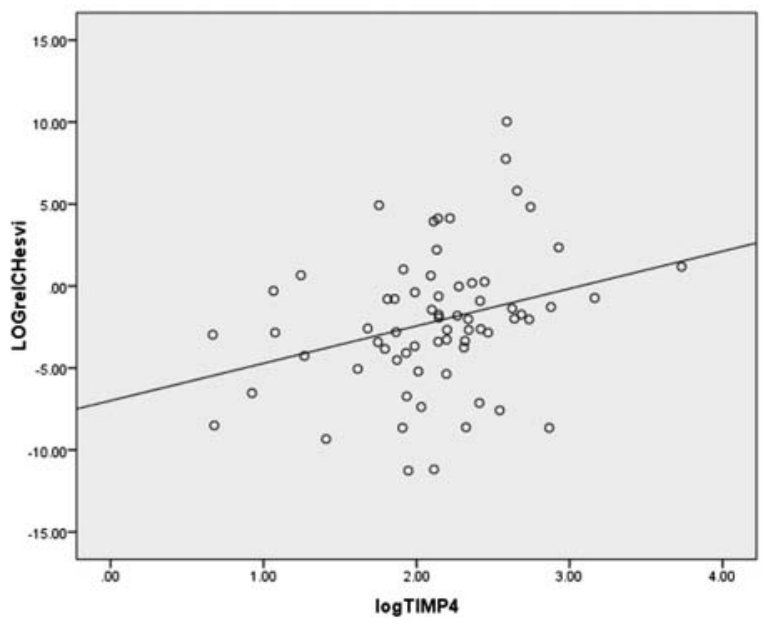

Figure 1

Conclusions/implications TIMP4 is an independent predictor of a change in LVESVI in patients with STEMI. Early TIMP4 measurement might select patients at risk of subsequent LV remodelling. NTproBNP is an independent predictor of EF\% and IS\%. 